\title{
Expression of thymidylate synthase (TS) and its prognostic significance in patients with cutaneous angiosarcoma
}

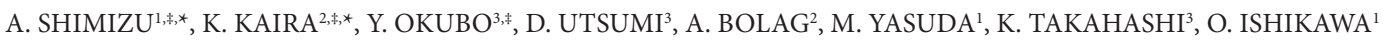 \\ ${ }^{1}$ Department of Dermatology and ${ }^{2}$ Department of Oncology Clinical Development, Gunma University Graduate School of Medicine; ${ }^{3}$ Department \\ of Dermatology, Graduate School of Medicine, University of the Ryukyus
}

*Correspondence: shimizuakira@gunma-u.ac.jp, kkaira1970@yahoo.co.jp

${ }^{*}$ Contributed equally to this work.

Received December 26, 2016 / Accepted April 9, 2017

\begin{abstract}
Cutaneous angiosarcoma (CA) is extremely rare, and little is known about the biological significance of possible biomarkers for chemotherapeutic agents. Thymidylate synthase (TS) is an attractive target for cancer treatment in various human neoplasms. It remains unclear whether the expression of TS is associated with the clinicopathological features of CA patients. The aim of this study was to elucidate the relationship between TS expression and the clinicopathological significance in CA patients. Fifty-one patients with CA were included in this study. TS expression and Ki-67 labeling index were examined using immunohistochemical analysis. TS was positively expressed in 39\% (20/51) of CA patients. No statistically significant prognostic factor was identified as a predictor of overall survival (OS) for all patients by univariate analysis, whereas a significant prognostic variable for progression free survival (PFS) was found to be the clinical stage. In addition, both univariate and multivariate analyses confirmed that positive expression of TS was a significant predictor of worse PFS in CA patients of clinical stage 1. Conclusion: Positive TS expression in CA was identified as a significant predictor of worse outcome in patients of clinical stage 1.
\end{abstract}

Key words: thymidylate synthase, prognosis, angiosarcoma, skin cancer, immunohistochemistry

Cutaneous angiosarcoma (CA) is a very rare sarcoma arising from the vascular endothelium, and can occur anywhere, although the head and neck are the most common primary sites. This aggressive sarcoma is associated with a poor prognosis, and five-year survival rates have been reported to be between $10 \%$ and $35 \%[1,2]$. An optimal therapeutic regimen for CA remains undefined. Systemic chemotherapy or radiotherapy has been applied where extensive or recurrent disease preclude complete resection, and several researchers have reported recently that taxane agents such as paclitaxel and docetaxel yielded promising outcomes in unresectable angiosarcoma $[3,4]$.

No suitable biomarkers have been identified to predict prognosis after any treatments against CA. Thymidylate synthase (TS) is an enzyme that catalyzes the methylation of deoxyuridine monophosphate (dUMP) to deoxythymidine monophosphate (dTMP) [5], and TS expression exhibits a significant positive correlation with tumor activity in many human cancers. In recent clinical studies, increased expression of TS was closely associated with tumor cell proliferation, pro- gressiveness, and survival rate $[6,7]$, and enhanced expression of TS in malignant melanoma revealed a significant association with angiogenesis, proliferation, and poor outcome [8]. TS has been identified as a target enzyme of various chemotherapeutic agents such as 5-fluorouracil (5-FU), a chemotherapeutic agent widely used to treat various human neoplasms, S-1 (Taiho Pharmaceutical Co. Ltd., Tokyo, Japan), and pemetrexed; S-1 and pemetrexed are used in standard chemotherapeutic regimens for lung cancer, and S-1 has been introduced against gastrointestinal cancers, breast cancer, pancreatic cancer, and head and neck cancers. Comprehensive analysis has revealed that low expression of TS was closely associated with better survival, compared with high expression of TS, and that patients with low TS expression in non-small cell lung cancers exhibited better outcome after TS-targeted treatments [9]. Moreover, in vitro study using sarcoma cell lines revealed inhibition of TS expression in cells in which tumor growth was suppressed by exposure to S-1 [10]. Although TS may also play a crucial role in the pathogenesis and tumor progression of skin neoplasms, little is known about the relationship between 
TS expression and CA. To elucidate the relationship between TS expression and patient outcome in CA, we conducted an immunohistochemical study.

\section{Patients and methods}

Patients. Fifty-one consecutive patients diagnosed definitively with CA at Gunma University Hospital and the University of the Ryukyus from October 1987 through September 2014 were included in this study. Fifty-one paraffin-embedded tissue samples were analyzed together with data from medical records obtained from both institutions. This study was approved by the institutional review board of Gunma University Hospital and the University of the Ryukyus (the Ethical Committee for Clinical Studies). The authors' approach to the evaluation and resection of these tumors has been described previously [11].

There is no well-defined clinical tumor staging formula accepted worldwide for CA. According to previous study [12], we classified CA patients into 3 stages: stage 1, patients with localized cutaneous tumors; stage 2 , patients with lymph node metastases; and stage 3 , patients with distant metastases. As an initial treatment, 8 patients were initially treated with surgical resection plus chemoradiotherapy, 6 patients surgical resection plus radiation, 2 patients surgical resection plus chemotherapy, 15 patients chemoradiotherapy, 12 patients radiation, 2 patients chemotherapy and 5 patients didn't receive any treatment. The majority of systemic chemotherapy consists of taxane-based regimens.

Immunohistochemical staining. The immunohistochemical staining for TS and Ki-67 was performed according to the procedures described in previous reports $[6-8,13]$. The following antibodies were used: a rabbit monoclonal antibody against TS (D5B3, Cell Signaling Technology; 1:200 dilution) and mouse monoclonal antibodies against Ki-67 (Dako, Glostrup, Denmark; 1:40 dilution). The expression of TS was considered positive if nucleus or cytoplasm staining was present, and the representative tumor area was identified using HE staining and immunohistochemical staining for CD34 or D2-40. The TS-positive tumor cells were counted in the tumor area at high magnification $(\times 400)$, and the percentage of TS-positive tumor cells was represented according to a semi-quantitative scoring method: $1,<10 \% ; 2,10-24 \% ; 3,25-50 \%$; and $4,51-75 \%$. Samples with a score of 2 or more (i.e., $\geq 10 \%$ TS-positive tumor cells) were graded as positive.

Analysis of Ki-67 expression was carried out as follows. A highly cellular area of the immunostained section was evaluated, and approximately 1000 nuclei were counted on each slide. All angiosarcoma cells with Ki-67 nuclear staining of any intensity were defined as having positive expression, and proliferative activity was assessed as the percentage of Ki-67-stained nuclei (Ki-67 labeling index) in the sample. The median value of the Ki-67 labeling index was determined, and the samples with a Ki-67 labeling index greater than the median value were defined as having high expression. Consequently, the tumors in which Ki-67-stained tumor cells made up more than $25 \%$ of the tumor (score 3 or 4 ) were graded as high. The sections were assessed using light microscopy in a blinded fashion by at least two of the authors.

Statistical analysis. Probability values of $<0.05$ indicated a statistically significant difference. The significance of the difference was determined using Fisher's exact test. The correlation between different variables was analyzed using the nonparametric Spearman's rank test. The Kaplan-Meier method was used to estimate survival as a function of time, and survival differences were analyzed by the log-rank test. Overall survival (OS) was determined as the time from definite diagnosis to death from any cause. Progression-free survival (PFS) was defined as the time between definite diagnosis and the first disease progression or death. Multivariate analyses were performed using a stepwise Cox proportional hazards model to identify independent prognostic factors. The statistical analysis was performed using GraphPad Prism 4 software (Graph Pad Software, San Diego, CA, USA) and JMP 8 (SAS Institute Inc., Cary, NC, USA) for Windows.

\section{Results}

Patients and pathological findings. Patient demographics based on TS expression are listed in Table 1. The median age

Table 1. Patient's demographics according to TS expression

\begin{tabular}{|c|c|c|c|c|c|}
\hline \multirow{2}{*}{ Variables } & & \multirow[b]{2}{*}{ Total $(n=51)$} & \multicolumn{3}{|c|}{ TS } \\
\hline & & & Positive $(n=20)$ & Negative (n=31) & p-value \\
\hline Age & $\leq 75 />75 \mathrm{yr}$ & $24 / 27$ & 11.9 & $13 / 18$ & 0,402 \\
\hline Gender & Male / Female & $35 / 16$ & 14.6 & 21.10 & $>0.999$ \\
\hline Primary site & Parietal / non-parietal & $26 / 25$ & 9.11 & $17 / 14$ & 0,572 \\
\hline Tumor size $(\mathbf{m m})$ & $\leq 45 />45 \mathrm{~mm}$ & $27 / 24$ & 13.7 & $14 / 17$ & 0,251 \\
\hline Clinical stage & $1 / 2$ or 3 & $38 / 13$ & 14.6 & 24.7 & 0,743 \\
\hline Lymph node metastasis & Yes / No & 3.42 & * $2 / 14$ & $* 1 / 28$ & 0,284 \\
\hline Distant metastasis & Yes / No & 10.40 & $* 5 / 14$ & 5.26 & 0,474 \\
\hline Ki-67 & High / Low & $27 / 24$ & 12.8 & $15 / 16$ & 0,566 \\
\hline
\end{tabular}

* These variables lack some data of patients 


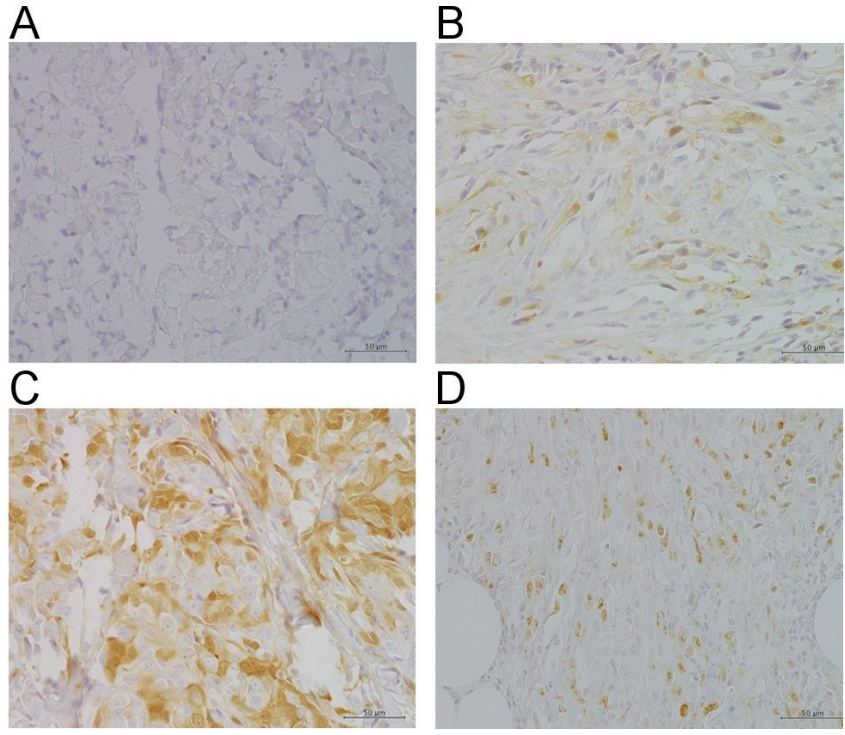

Figure 1. Immunohistochemical staining as representative pictures in cutaneous angiosarcoma. Positive staining of TS expression showing the cytoplasma and nuclei of cutaneous angiosarcoma. The scoring of TS Immunostaining was grade 1 (A), grade 2 (B) and grade 3 (C). The representative imaging of $\mathrm{Ki}-67$ (D) was presented.

was 76 years (57-86 years). The primary site of the disease was the scalp in 34 patients, face in 13, and neck in 4 . Clinical staging was as follows: stage 1, 38 patients; stage 2, 2 patients; and stage 3, 11 patients. Surgical resection was applied in 16 patients, radiotherapy in 44 , and chemotherapy in 30 . Docetaxel was administered in 25 patients. The median follow-up period was 370 days (56-2800 days).

Representative samples with TS expression levels of scores 1-3 are shown in Figure 1A-C. TS expression was observed predominantly in the cytoplasm of angiosarcoma cells. TS was positively expressed in 39\% (20/51) of samples, and the average score for TS was $1.6 \pm 0.8$. The percentage samples with TS scores of $1,2,3$, and 4 were $61 \%(31 / 51), 22 \%(11 / 51), 17 \%(9 / 51)$, and $0 \%(0 / 51)$, respectively. The median Ki-67 labeling index in all CA specimens was 9\% (range, $0-51 \%$ ), and the value of $9 \%$ was chosen as the cut-off point. Hence, a high labeling index for Ki67 was shown in 50\% (25/51) of samples. Representative positive immunohistochemical staining for Ki-67 is shown in Figure 1D.

Relationship between TS expression and assorted variables. The correlations between the expression level of TS and assorted variables were analyzed using Spearman's rank test. The TS expression did not yield a statistically significant correlation with Ki-67 $(\mathrm{r}=-0.049, \mathrm{P}=0.727)$ or tumor size $(\mathrm{r}=$ $-0.029, \mathrm{P}=0.835$ ).

Survival analysis. Thirty-nine patients developed recurrence after initial treatment, and 40 patients died due to the progression of primary disease. The median survival times (MSTs) of OS and PFS for all patients were 448 days and 270 days, respectively. The one-year survival rates for OS and PFS for all patients were $55 \%$ and $37 \%$, respectively. The results of univariate and multivariate analyses for all patients are listed in Table 2. No statistically significant prognostic factor was identified as a predictor of OS and PFS (Figure 2A, B) for all patients by univariate analysis,
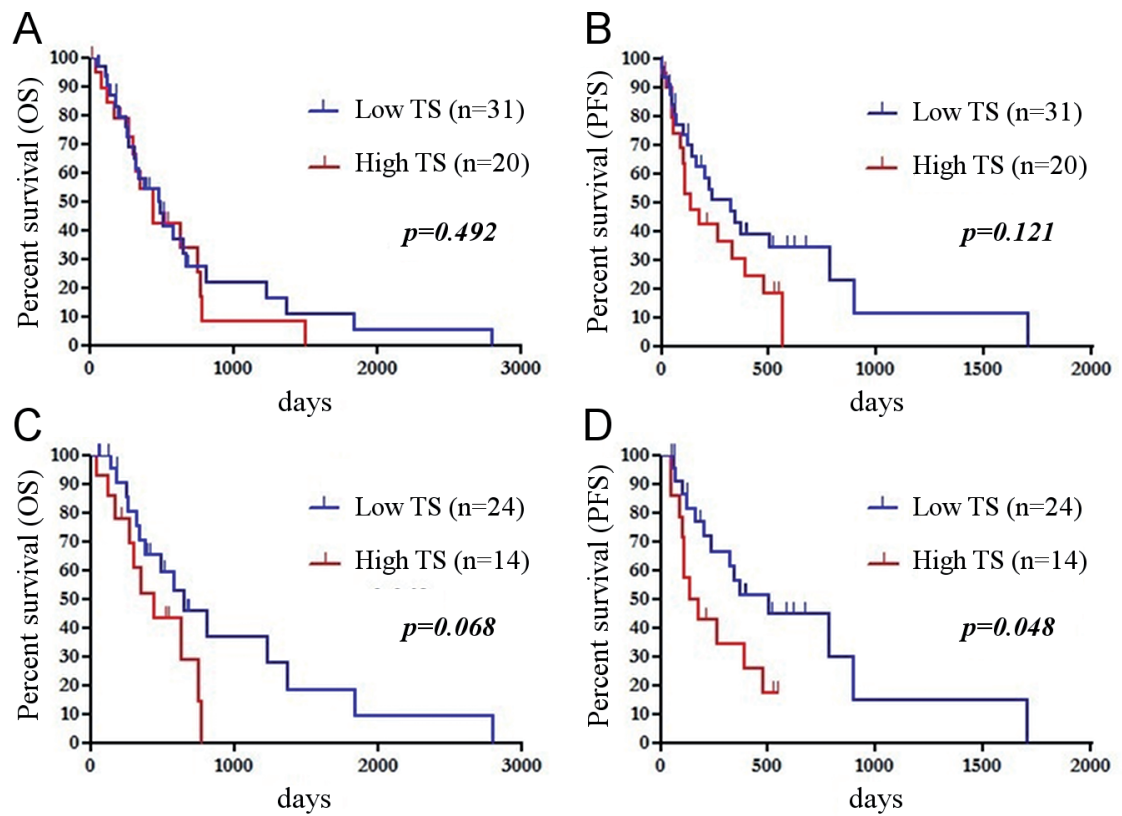

Figure 2. Kaplan-Meier survival curve of OS and PFS according to the expression of TS. No statistically significant difference in the OS (A) and PFS (B) was recognized between all patients with positive and negative TS expression. In clinical stage I, there was no difference of OS (C) between patients with positive and negative TS expression, a statistically significant difference in PFS (D) was observed. 
Table 2. Univariate and multivariate survival analysis in all patients

\begin{tabular}{|c|c|c|c|c|c|c|c|c|c|c|c|}
\hline & \multirow{3}{*}{ Variables } & \multicolumn{5}{|c|}{ Overall survival } & \multicolumn{5}{|c|}{ Progression-free survival } \\
\hline & & \multicolumn{2}{|c|}{ Univariate } & \multicolumn{3}{|c|}{ Multivariate } & \multicolumn{2}{|c|}{ Univariate } & \multicolumn{3}{|c|}{ Multivariate } \\
\hline & & MST (days) & $P$-value & HR & $95 \% \mathrm{CI}$ & $P$-value & MST & $P$-value & HR & $95 \% \mathrm{CI}$ & $P$-value \\
\hline Age & $\leq 75 />75 \mathrm{yr}$ & $356 / 495$ & 0,571 & 1,16 & $0.816-1.666$ & 0,403 & $141 / 328$ & 0,706 & 1,06 & $0.750-1.502$ & 0,738 \\
\hline Gender & Male / Female & $356 / 583$ & 0,235 & 1,19 & $0.805-1.836$ & 0,386 & $165 / 477$ & 0,016 & 1,51 & $0.988-2.445$ & 0,056 \\
\hline Primary site & Parietal / non-parietal & $387 / 583$ & 0,22 & & & & $200 / 235$ & 0,808 & & & \\
\hline Tumor size $(\mathbf{m m})$ & $\leq 45 />45 \mathrm{~mm}$ & $518 / 326$ & 0,643 & & & & $328 / 165$ & 0,494 & & & \\
\hline Clinical stage & $1 / 2$ or 3 & $583 / 326$ & 0,087 & 1,27 & $0.841-1.898$ & 0,247 & $342 / 50$ & $<0.001$ & 1,61 & $1.065-2.388$ & 0,024 \\
\hline TS & Positive / Negative & $441 / 495$ & 0,492 & 1,02 & $0.703-1.467$ & 0,899 & $135 / 321$ & 0,121 & 1,23 & $0.842-1.778$ & 0,284 \\
\hline Ki-67 & High / Low & $356 / 583$ & 0,11 & & & & $175 / 388$ & 0,071 & & & \\
\hline
\end{tabular}

Abbreviation: HR, hazard ratio; 95\% CI, 95\% confidence interval.

whereas a significant prognostic variable for PFS was found to be the clinical stage (Table 2). The multivariate analysis confirmed the clinical stage as an independent prognostic factor for poor PFS (Table 2). Next, we analyzed the prognostic significance of TS expression in 38 patients of clinical stage 1 (Table 3 ), in which the number of samples with positive and negative TS expression was 14 and 24, respectively. By univariate analysis, the expression of TS was identified as having borderline significance $(\mathrm{P}=0.068)$ for predicting poor OS (Figure 2C), but multivariate analysis could not confirm the TS expression to be an independent predictor of worse OS (Table 3). However, the univariate analysis for PFS identified male gender and positive TS expression as significant factors for worse prognosis (Figure 2D). Multivariate analysis confirmed that male gender $(\mathrm{P}=0.001)$ and positive TS expression $(\mathrm{P}=0.004)$ were independent prognostic variables to predict poor PFS (Table 3).

\section{Discussion}

This is the first study demonstrating the clinicopathological features of TS expression in patients with CA. We found that TS was expressed in 39\% of CA specimens and that positive expression of TS was a significant prognostic predictor for PFS in CA patients of clinical stage 1 .

Clinically, TS is an important enzyme target for moleculartargeting drugs aimed at suppressing the tumor growth of various neoplasms $[8,14,15]$. The expression level of TS differs markedly among neoplasms. The positive rates of TS expression were $86 \%$ in lung squamous cell carcinoma, $51 \%$ in lung adenocarcinoma, $35 \%$ in lung sarcoma, $47 \%$ in mesothelioma, and $26 \%$ in cutaneous malignant melanoma [6-8]. TS-targeting agents such as S-1 and pemetrexed are recognized to be effective for patients with lung cancer, contributing to improved survival time $[14,15]$. Apoptosis, DNA damage, and S-phase cell cycle arrest by TS inhibitors have been considered possible mechanisms by in vitro studies [16-18].

It has been demonstrated that enhanced expression of TS is closely linked to an aggressive phenotype, tumor proliferation, and poor prognosis in malignant tumors [19]. In a previous report, the expression of TS was linked to the Akt/mTOR pathway in cancers, and the activation of this pathway may play a crucial role in the overexpression of TS [20]. Although some studies have demonstrated a positive correlation between TS expression and Ki-67 in cutaneous malignant melanoma and non-small cell lung cancer $[8,19]$, TS expression did not correlate with Ki-67 expression level in CA. It is unclear why TS expression did not correlate with Ki-67 expression in the present study.

A recent meta-analysis showed that TS was identified as a significant predictor for pemetrexed as a TS inhibitor [21]. Takeda et al. reported that low expression of TS was associated with a favorable response and better prognosis in patients with

Table 3. Univariate and multivariate survival analysis in patients of clinical stage 1

\begin{tabular}{|c|c|c|c|c|c|c|c|c|c|c|c|}
\hline & \multirow{3}{*}{ Variables } & \multicolumn{5}{|c|}{ Overall survival } & \multicolumn{5}{|c|}{ Progression-free survival } \\
\hline & & \multicolumn{2}{|c|}{ Univariate } & \multicolumn{3}{|c|}{ Multivariate } & \multicolumn{2}{|c|}{ Univariate } & \multicolumn{3}{|c|}{ Multivariate } \\
\hline & & MST(days) & $P$-value & HR & $95 \% \mathrm{CI}$ & $P$-value & MST & $P$-value & HR & $95 \% \mathrm{CI}$ & $P$-value \\
\hline Age & $\leq 75 />75 \mathrm{yr}$ & $356 / 652$ & 0,992 & 1,11 & $0.705-1.751$ & 0,657 & $264 / 388$ & 0,532 & 1,04 & $0.672-1.614$ & 0,845 \\
\hline Gender & Male / Female & $356 / 652$ & 0,25 & 1,37 & $0.882-2.224$ & 0,166 & $200 / 1700$ & 0,02 & 2,26 & $1.352-4.153$ & 0,001 \\
\hline Primary site & Parietal / non-parietal & $387 / 638$ & 0,321 & & & & $321 / 388$ & 0,568 & & & \\
\hline Tumor size $(\mathbf{m m})$ & $\leq 45 />45 \mathrm{~mm}$ & $638 / 387$ & 0,568 & & & & $342 / 200$ & 0,889 & & & \\
\hline TS & Positive / Negative & $448 / 652$ & 0,068 & 1,54 & $0.981-2.412$ & 0,059 & $135 / 502$ & 0,048 & 2,02 & $1.249-3.330$ & 0,004 \\
\hline Ki-67 & High / Low & $387 / 652$ & 0,344 & & & & $235 / 477$ & 0,237 & & & \\
\hline
\end{tabular}

Abbreviation: HR, hazard ratio; 95\% CI, 95\% confidence interval. 
lung cancer who received regimens including S-1 [22]. In contrast, strong TS expression was correlated with worse outcome in these patients. These results suggest that TS inhibitors may be more effective for lung cancers with low TS expression, although the precise mechanism is unknown. Although further investigation is necessary, inhibitors of TS may be worth investigating as possible therapeutic agents in CA.

Recently, we have already published the clinicopathological significance of the other markers including programmed death-ligand 1 (PD-L1) and CD98 for the patients with CA $[11,23]$. The positive expression of $\mathrm{PD}-\mathrm{L} 1$ was identified as an independent prognostic factor to predict worse survival in the patients with CA, and PD-L1 expression was significantly associated with tumor cell proliferation [11]. Moreover, we found that CD98 linked to amino acid transporter is also important as a significant predictor in the patients with CA [23]. Especially, the low expression of CD98 was significantly associated with distant metastasis and shorter survival. Considering the results of these two markers, PD-L1 and CD98 seemed to be better to predict the outcome after any treatment against the patients with CA rather than TS. However, our small-sample size may bias the interpretation of prognosis after treatment according to the expression of different kinds of biomarkers. Further study is warranted to compare TS from the other biomarkers about the prognostic significance of CA patients.

There are several limitations in the current study. Our sample size was limited, which might have affected our study results. As CA is extremely rare and its incidence differs depending on race or country, it may be difficult to perform a large-scale study. Next, we could not perform in vitro analysis to elucidate the relationship between the expression level of TS before and after TS inhibitor treatment. TS inhibitors such as pemetrexed and 5-FU have been widely administered against various human cancers; however, it remains unclear whether the inhibitors of TS could successfully suppress the tumor growth of CA. Further investigation is warranted to confirm the results of our study by using a validation cohort and investigating the association between the expression level of TS and the therapeutic efficacy of TS-targeted agents.

In conclusion, TS was frequently expressed in CA (20/51, $39 \%)$. Our study demonstrated that positive TS expression was identified as a significant predictor of worse outcome. Further study should focus on translational research to determine whether TS inhibitors are potential therapeutic agents for effective targeting in CA depending on the expression level of TS.

Acknowledgments: We thank Ms. Yuka Matsui (Gunma University), Ms. Ayako Nakamura, and Ms. Ritsuko Tokumon (University of the Ryukyus) for their technical assistance during the manuscript preparation. We also thank Ms. Tomoko Okada (Gunma University) for her help in data collection and technical assistance and Drs. Ryoko Awazawa, Takuya Miyagi, and Sayaka Yamaguchi (University of the Ryukyus) for their helpful guidance and assistance in data collection.

\section{References}

[1] MENDENHALL WM, MENDENHALL CM, WERNING JW, REITH JD, MENDENHALL NP. Cutaneous angiosarcoma. Am J Clin Oncol 2006; 29: 524-528. https://doi. org/10.1097/01.coc.0000227544.01779.52

[2] GUADAGNOLO BA, ZAGARS GK, ARAUJO D, RAVI V, SHELLENBERGER TD et al. Outcomes after definitive treatment for cutaneous angiosarcoma of the face and scalp. Head Neck 2011; 33: 661-667. https://doi.org/10.1002/hed.21513

[3] PENEL N, BUI BN, BAY JO, CUPISSOL D, RAY-COQUARD I et al. Phase II trial of weekly paclitaxel for unresectable angiosarcoma: the ANGIOTAX study. J Clin Oncol 2008; 26: 5269-5274. https://doi.org/10.1200/JCO.2008.17.3146

[4] NAGANO T1, YAMADA Y, IKEDA T, KANKI H, KAMO $T$ et al Docetaxel a therapeutic option in the treatment of cutaneous angiosarcoma. Cancer 2007; 110: 648-651. https:// doi.org/10.1002/cncr.22822

[5] DANENBERG PV. Thymidylate synthetase - a target enzyme in cancer chemotherapy. Biochim Biophys Acta 1997; 473: 73-92.

[6] KAIRA K, YAMAMOTO N, ENDO M, KENMOTSU H, NAITO T et al. 18F-FDG uptake on PET is a predictive marker of thymidylate synthase expression in patients with thoracic neoplasms. Oncol Rep 2014; 31: 209-215. https:// doi.org/10.3892/or.2013.2816

[7] KAIRA K, OHDE Y, NAKAGAWA K, OKUMURA T, MURAKAMI $\mathrm{H}$ et al. Thymidylate synthase expression is closely associated with outcome in patients with pulmonary adenocarcinoma. Med Oncol 2012; 29: 1663-1672. https:// doi.org/10.1007/s12032-011-0069-8

[8] SHIMIZU A, KAIRA K, YASUDA M, ASAO T, ISHIKAWA O. Prognostic significance of thymidylate synthase (TS) expression in cutaneous malignant melanoma. Neoplasma 2016; 63: 282-287. https://doi.org/10.4149/215 150714N389

[9] LIU Q, YU Z, XIANG Y, WU N, WU L et al. Prognostic and predictive significance of thymidylate synthase protein expression in non-small cell lung cancer: a systematic review and meta-analysis. Cancer Biomark 2015; 15: 65-78. https://doi. org/10.3233/CBM-140432

[10] YURIMOTO S, MIYAKAWA A, OKUZAWA A, SAKAMOTO $S$, SAKAMOTO Ket al. Enhancement of the anti-tumor activity of S-1 by low-dose cisplatin in mice bearing the sarcoma-180 model. Anticancer Drugs 2005; 16: 1109-1114. https://doi. org/10.1097/00001813-200511000-00010

[11] A. SHIMIZU, K. KAIRA, Y. OKUBO, D. UTSUMI, M. YASUDA et al. Positive PD-L1 expression predicts worse outcome in cutaneous angiosarcoma. J Glob Oncol 2016, in press. https://doi.org/10.1200/JGO.2016.005843

[12] FUJII H, ARAKAWA A, UTSUMI D, SUMIYOSHI S, YAMAMOTO Y et al. CD8+ tumor-infiltrating lymphocytes at primary sites as a possible prognostic factor of cutaneous angiosarcoma. Int J Cancer 2014; 134: 2393-2402. https://doi. org/10.1002/ijc.28581

[13] SHIMIZU A, KAIRA K, KATO M, YASUDA M, TAKAHASHI A et al. Prognostic significance of L-type amino acid transporter 1 (LAT1) expression in cutaneous melanoma. 
Melanoma Res 2015; 25: 399-405. https://doi.org/10.1097/ CMR.0000000000000181

[14] OKAMOTO I, YOSHIOKA H, MORITA S, ANDO M, TAKEDA K et al. Phase III trial comparing oral S-1 plus carboplatin with paclitaxel plus carboplatin in chemotherapy-naïve patients with advanced non-small-cell lung cancer: results of a west Japan oncology group study. J Clin Oncol 2010; 28: 5240-5246. https://doi.org/10.1200/JCO.2010.31.0326

[15] SCAGLIOTTI GV, PARIKH P, VON PAWEL J, BIESMA B, VANSTEENKISTE $J$ et al. Phase III study comparing cisplatin plus gemcitabine with cisplatin plus pemetrexed in chemotherapy-naive patients with advanced-stage non-smallcell lung cancer. J Clin Oncol 2008; 26: 3543-3551. https:// doi.org/10.1200/JCO.2007.15.0375

[16] GIUDICE S, BENASSI L, BERTAZZONI G, COSTI MP, GELAIN A et al. New thymidylate synthase inhibitors induce apoptosis in melanoma cell lines. Toxicol In Vitro 2007; 21: 240-248. https://doi.org/10.1016/j.tiv.2006.09.023

[17] GIUDICE S, BENASSI L, BERTAZZONI G, VERATTI E, MORINI D et al. Biological evaluation of MR36, a novel non-polyglutamatable thymidylate synthase inhibitor that blocks cell cycle progression in melanoma cell lines. Invest New Drugs 2012; 30: 1484-1492. https://doi.org/10.1007/ s10637-011-9733-2

[18] BUQUE A, MUHIALDIN JSH, MUNOZ A, CALVO B, CARRERA $S$ et al. Molecular mechanism implicated in Pemetrexed-induced apoptosis in human melanoma cells. Mol Cancer 2012; 11:25. https://doi.org/10.1186/1476-4598-11-25
[19] NAKAGAWA T, OTAKE Y, YANAGIHARA K, MIYAHARA $\mathrm{R}$, ISHIKAWA $\mathrm{S}$ et al. Expression of thymidylate synthase is correlated with proliferative activity in non-small cell lung cancer (NSCLC). Lung Cancer 2004; 43: 145-149. https://doi. org/10.1016/j.lungcan.2003.09.004

[20] OKABE T, OKAMOTO I, TSUKIOKA S, UCHIDA J, IWASA T et al. Synergistic antitumor effect of S- 1 and the epidermal growth factor receptor inhibitor gefitinib in nonsmall cell lung cancer cell lines: role of gefitinib-induced down-regulation of thymidylate synthase. Mol Cancer Ther 2008; 7: 599-606. https://doi.org/10.1158/1535-7163.MCT07-0567

[21] WANG L, WANG R, PAN Y, SUN Y, ZHANG J et al. The pemetrexed-containing treatments in the non-small cell lung cancer is -/low thymidylate synthase expression better than + / high thymidylate synthase expression: a meta-analysis. BMC Cancer 2014; 14: 205. https://doi.org/10.1186/1471-2407-14$\underline{205}$

[22] TAKEDA M, OKAMOTO I, HIRABAYASHI N, KITANO M, NAKAGAWA K. Thymidylate synthase and dihydropyrimidine dehydrogenase expression levels are associated with response to S-1 plus carboplatin in advanced non-small cell lung cancer. Lung Cancer 2011: 73: 103-109. https://doi. org/10.1016/j.lungcan.2010.10.022

[23] SHIMIZU A, KAIRA K, OKUBO Y, UTSUMI D, YASUDA $M$ et al. Prognostic impact of LAT1 and CD98 expression in cutaneous angiosarcoma. Neoplasma 2017; 64: 283-288 https://doi.org/10.4149/neo 2017216 